\title{
A rare variation of the glossopharyngeal nerve
}

\author{
Yusra Mansour, Randy Kulesza \\ Department of Anatomy, Lake Erie College of Osteopathic Medicine, Erie, PA, USA
}

\begin{abstract}
The glossopharyngeal nerve (CN IX) provides innervation to the parotid gland, carotid body/sinus, mucosa of the middle ear, tongue and oropharynx and the stylopharyngeus muscle. The vagus nerve provides innervation to the remaining skeletal muscle of the pharynx. CN IX contributes to the pharyngeal plexus and normally provides innervation to the mucosa of the oropharynx. Herein, we describe a previously undescribed variation of CN IX. CN IX was observed to enter the pharyngeal wall but instead of forming terminal branches in the tonsillar fossa, CN IX descended along the posterior wall between the mucosa and pharyngeal constrictors to the esophagus. This unusual branch of CN IX gave rise to numerous branches along the pharynx but did not intermingle with laryngeal branches from the vagus nerve. From this dissection, we developed innervation maps of the pharynx and propose a central miswiring mechanism for this unusual variation.
\end{abstract}

Key words: Cranial nerve, Autonomic, Pharynx pathways

Received January 18, 2021; Revised February 26, 2021; Accepted March 12, 2021

\section{Introduction}

The glossopharyngeal nerve (CN IX), includes both sensory and motor axons and emerges from the rostral medulla as several rootlets along the postolivary fissure, between the facial and vagus nerves. CN IX provides innervation to the external ear, parotid gland, mucosa of the tympanic cavity and mastoid sinuses, tongue, oropharynx, and carotid body/ sinus.

Efferent axons in CN IX are derived from the nucleus ambiguous (NA) and the inferior salivatory nucleus (ISN). The NA includes lower motor neurons that provide innervation to the stylopharyngeus muscle. The ISN provides preganglionic parasympathetic innervation to scattered ganglion cells in the tympanic cavity [1] and otic ganglion. There are two sensory ganglia associated with CN IX: the superior and inferior (petrosal). Together, these sensory ganglia include

\section{Corresponding author:}

Randy Kulesza (iD

Department of Anatomy, Lake Erie College of Osteopathic Medicine, Erie, PA 16509, USA

E-mail: rkulesza@lecom.edu pseudounipolar cell bodies that provide general somatic, visceral and special visceral innervation across the territory of CN IX. These primary sensory neurons project to the spinal trigeminal nucleus (STN) or solitary nuclei (SN). Axons reaching the STN convey pain and thermal sense from the external ear. Axons reaching the rostral SN convey gustatory sense from the posterior one-third of the tongue; those reaching more caudal SN relay visceral sensory information from the pharynx, middle ear, oral cavity and carotid sinus/ body.

CN IX sends thin communicating branches to the superior cervical ganglion, the facial nerve and a branch that joins the auricular branch of the vagus nerve $(\mathrm{CN} \mathrm{X})$-this combined auricular branch traverses the mastoid canaliculus and provides cutaneous sensory innervation to a small region of the external ear. Besides these smaller communicating branches, CN IX has six main branches [2]:

1. The tympanic nerve (nerve of Jacobson) arises from the inferior ganglion, traverses the tympanic canaliculus and provides innervation to the middle ear cavity, mastoid air cells and auditory tube via the tympanic plexus. Along the superior aspect of the tympanic cavity, the tympanic plexus converges to form the lesser petrosal nerve which provides preganglionic parasympathetic 


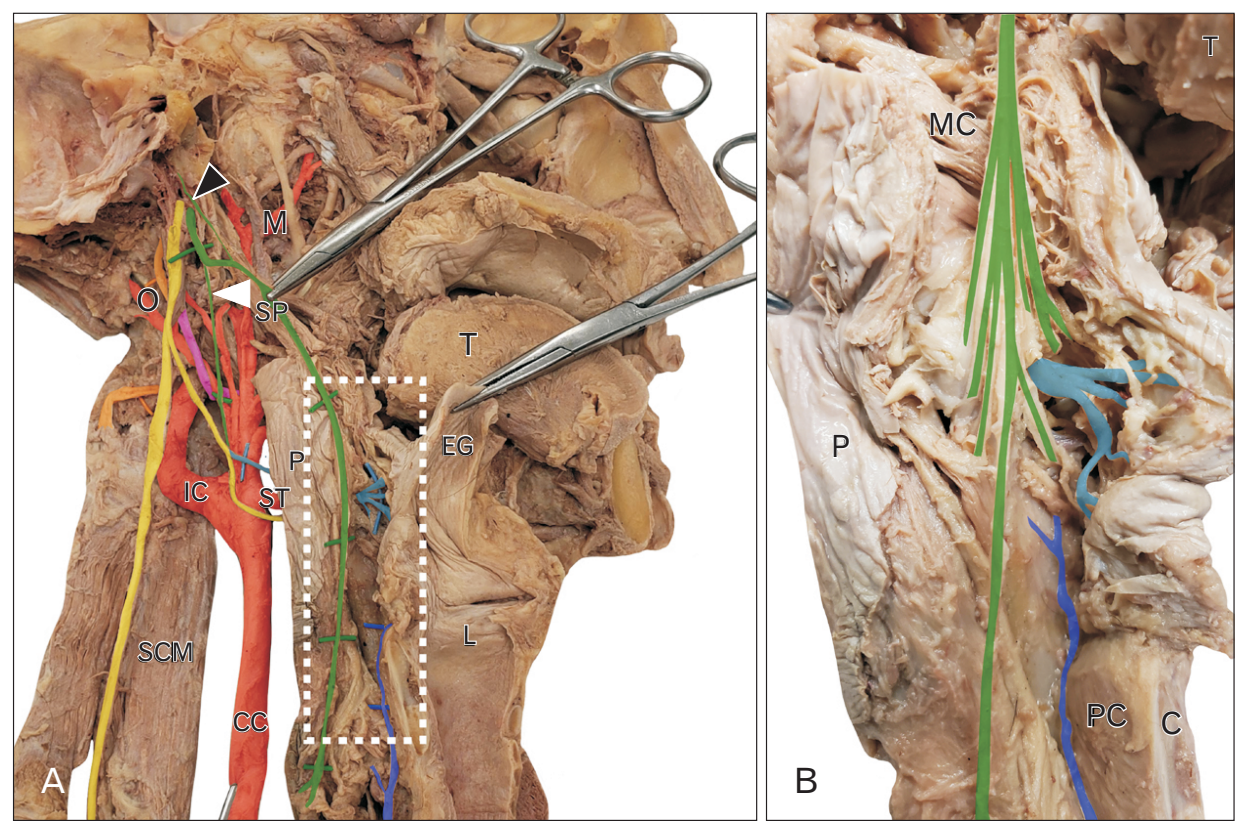

Fig. 1. Dissection of CN IX. (A) is the dissection of CN IX (green) and X (yellow). CN IX emerges from the skull and gives off a tympanic nerve (black arrowhead) and sinus nerve (solid arrowhead). CN IX sits on the stylopharyngeus muscle (SP) and enters the pharyngeal wall (P) deep to the middle constrictor muscle (MC). CN IX continues to descend along the posterior wall of the pharynx between the mucosa and muscle layers. The region indicated by the white rectangle is enlarged in (B) and demonstrates the numerous branches arising from CN IX in this specimen, distinct from the internal laryngeal nerve (cyan) and recurrent laryngeal nerve (blue). C, cricoid cartilage; CC, common carotid; EG, epiglottis; IC, internal carotid artery; L, larynx; L, lingual artery; M, maxillary artery; MC, middle constrictor; O, occipital artery; P, pharynx; PC, posterior cricoarytenoid; SCM, sternocleidomastoid; SP, stylopharyngeus; ST, superior thyroid artery; T, tongue.
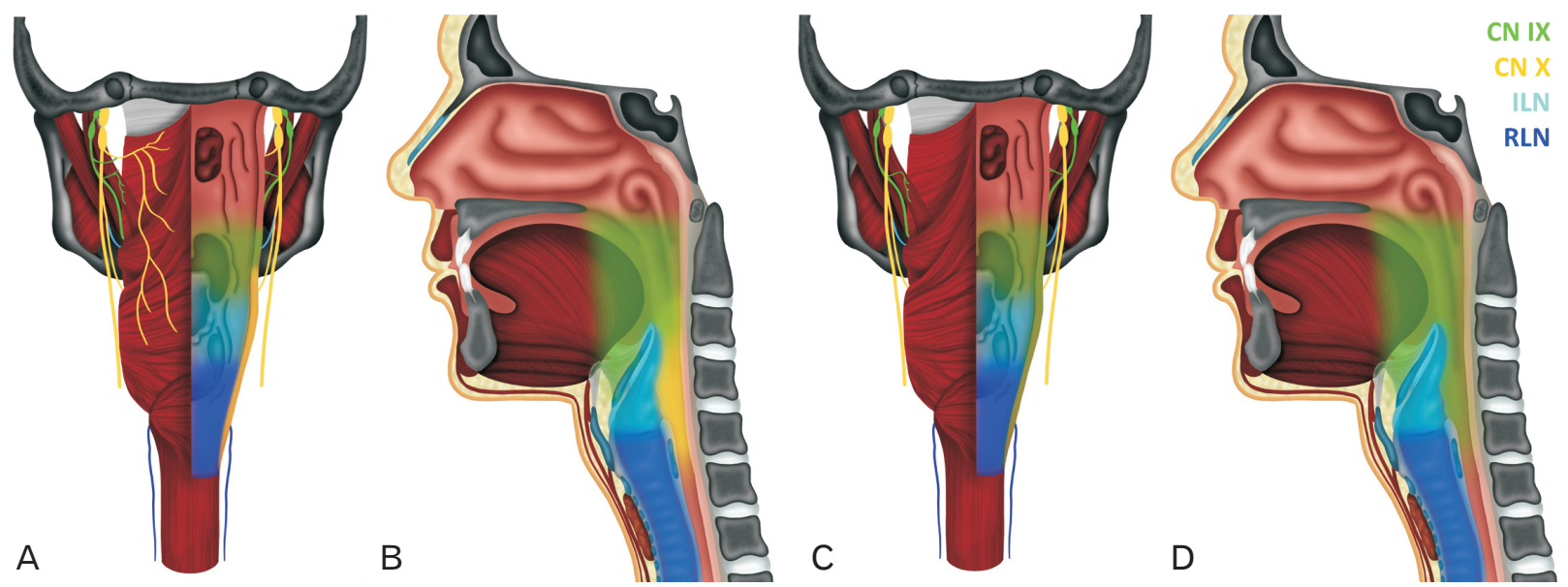

Fig. 2. Distribution of CN IX. Posterior (A) and midsagittal (B) show the normal distribution of CN IX (green) and X (yellow). Pharyngeal and tonsillar branches from CN IX supply the oropharynx and pharyngeal branches from CN X supply the hypopharynx. The ILN (cyan) and RLN (blue) provide innervation to the larynx. In the dissected specimen (C, D), CN IX appears to cover the entire posterior pharyngeal wall. ILN, internal laryngeal nerve; RLN, recurrent laryngeal nerve.

innervation to the otic ganglion. Post-synaptic neurons in the otic ganglion travel along the auriculotemporal nerve to distribute to the parotid gland.

2. The carotid sinus nerve (of Herring) arises inferior to the petrosal ganglion and provides sensory innervation to the carotid body and sinus.

3. A motor branch supplying the stylopharyngeus muscle. 4. Pharyngeal branches that join with pharyngeal branches 

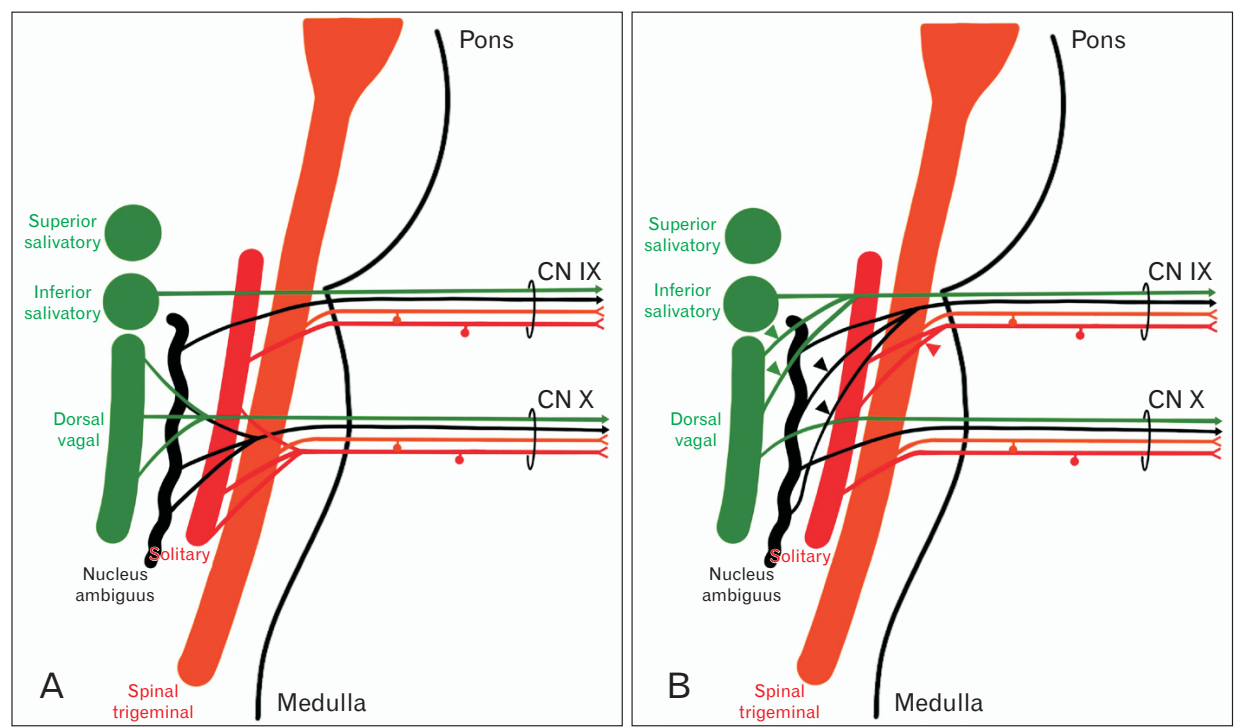

Fig. 3. Formation of CN IX and X. (A) A wiring diagram showing the brainstem nuclei associated with CN IX and X. (B) The proposed wiring pattern for CN IX in this subject. Since CN IX innervates the entire posterior pharyngeal wall, it takes a more extensive contribution from the ISN, NA and caudal SN. ISN, inferior salivatory nucleus; NA, nucleus ambiguus; SN, solitary nucleus. from CN X.

5. Tonsillar branches that provide visceral sensory innervation to the palatine tonsil, soft palate and faucial pillars.

6 . The terminal, lingual branch that provides sensory innervation to the posterior one-third of the tongue.

The pharyngeal branch from CN X emerges from the nodose (inferior) ganglion of $\mathrm{CN} \mathrm{X}$ and lies on the posterior (external) aspect of the middle pharyngeal constrictor muscle. The pharyngeal branch from $\mathrm{CN} X$ is joined by branches from the sympathetic chain and a pharyngeal branch from CN IX-together, this network forms the pharyngeal plexus. This plexus courses along the posterior aspect of the pharynx and innervates all muscles of the pharynx and soft palate except the stylopharyngeus and tensor veli palatini muscles. Additionally, the pharyngeal plexus provides autonomic innervation to the mucosa of the pharynx and esophagus.

While there are a number of reported variants involving branches of CN IX, there are only few reports of variants involving pharyngeal or motor branches. Specifically, CN IX may provide an additional contribution to the superior laryngeal nerve from $\mathrm{CN}$ X [3]. Also, the pharyngeal plexus may issue a middle laryngeal nerve that supplies the cricothyroid muscle, pierces the cricothyroid membrane and supplies the mucosa of the lower aspect of the larynx [3-5]. However, our research indicates this middle laryngeal nerve was identified in rabbits and/or dogs but not humans [6]. Regardless, variations of the pharyngeal distribution of CN IX in humans appears to be extremely rare.

\section{Case Report}

During dissection of the skull base exposing the jugular foramen and exit of CN IX-XII a peculiar innervation of the pharyngeal wall was discovered. CN IX emerged from the jugular foramen distinct from $\mathrm{CN}$ X (Fig. 1, green). In this subject, CN IX gave rise to a slender branch directed superiorly towards the skull base (tympanic nerve; Fig. 1A, black arrowhead), the sinus nerve (Fig. 1A, solid arrowhead) and motor branches to the stylopharyngeus muscle (Fig. 1). However, after slipping between the superior and middle constrictor muscles, CN IX continued to descend between the pharyngeal mucosa and constrictor muscles all the way to the esophagus (Fig. 1). This long pharyngeal variant of $\mathrm{CN}$ IX gave rise to a number of branches distributing to the mucosa and muscle wall (Fig. 1B, green) and coursed along the posterior pharyngeal wall of the oro- and laryngopharynx and remained distinct from the internal laryngeal nerve (Fig. 1, cyan) and the recurrent laryngeal nerve (Fig. 1, blue). No pharyngeal branches were found arising from the vagus nerve in this subject (Fig. 2C).

\section{Discussion}

We were interested in mapping the innervation of the pharynx in this subject and comparing it to the normal pattern. Fig. 2 shows the normal branching pattern of CN IX (green) and X (yellow) from posterior (Fig. 2A) and midsagittal (Fig. 2B) views. In the specimen, the $\mathrm{CN}$ IX variant appears to cover the normal oropharyngeal territory but also 
takes over the entire vagal pharyngeal territory (Fig. $2 \mathrm{C}$ and $\mathrm{D}$, green). We were also interested in how this peripheral innervation pattern might be arranged relative to the brainstem nuclei. The normal wiring of CN IX and $\mathrm{X}$ are shown in Fig. 3A. In the variant pattern (Fig. 3B), we believe that CN IX takes additional axons from the NA (black arrowheads), the dorsal vagal nucleus (green arrowheads) and caudal SN (red arrowheads). Based on the distribution of this variant nerve, we do not believe there are any additions to the number of axons carrying taste or somatic sensation.

This case report describes a previously undescribed variant where CN IX appears to cover the entire vagal innervation of the posterior pharyngeal wall. Because of the proximity of their nuclei in the medulla and their close relationship in the jugular foramen and skull base, CN IX and X are often injured together and their signs and symptoms are closely linked [2]. However, we suspect that in cases of glossopharyngeal neuralgia a variant such as described here would result in more widespread and more severe symptoms [7].

\section{ORCID}

Yusra Mansour: https://orcid.org/0000-0002-6421-1224

Randy Kulesza: https://orcid.org/0000-0001-8589-1532

\section{Author Contributions}

Conceptualization: YM. Data acquisition: YM. Data analysis or interpretation: YM, RK. Drafting of the manuscript: YM, RK. Critical revision of the manuscript: YM, RK. Approval of the final version of the manuscript: all authors.

\section{Conflicts of Interest}

No potential conflict of interest relevant to this article was reported.

\section{References}

1. Crosby EC, Humphrey T, Lauer EW. Correlative anatomy of the nervous system. New York: Macmillan Co; 1962.

2. Standring S. Gray's anatomy: the anatomical basis of clinical practice. 41st ed. Philadelphia: Elsevier; 2016.

3. Bergman RA, Thompson SA, Afifi AK. Compendium of human anatomic variation: text, atlas, and world literature. Baltimore: Urban \& Schwarzenberg; 1988.

4. Quain J, Schaefer EA, Thane GD. Quain's Elements of anatomy. 10th ed. London: Longmans, Green; 1897.

5. Tubbs RS, Shoja MM, Loukas M. Bergman's comprehensive encyclopedia of human anatomic variation. Hoboken: Wiley Blackwell; 2016.

6. Exner ID. Innervation des Kehlkopfes. Wien: Sitzungsber. d. Akad. d. Wissensch; 1884.

7. Singh PM, Kaur M, Trikha A. An uncommonly common: glossopharyngeal neuralgia. Ann Indian Acad Neurol 2013;16:1-8. 